\title{
Evaluation of antigenic variations between two virulent toxoplasma strains
}

\section{Correspondence \\ Songul Bayram Delibas \\ songul.bdelibas@deu.edu.tr}

Received 8 May 2006

Accepted 14 June 2006

\author{
Songul Bayram Delibas, ${ }^{1}$ Hatice Ertabaklar ${ }^{2}$ and Sema Ertug ${ }^{2}$ \\ ${ }^{1}$ Dokuz Eylül University, Medical Faculty, Department of Parasitology, 35340 Inciralti, Izmir, \\ Turkey \\ ${ }^{2}$ Adnan Menderes University, Medical Faculty, Department of Parasitology, Aydin, Turkey
}

\section{INTRODUCTION}

Toxoplasma gondii is an obligate intracellular protozoan parasite capable of infecting all species of mammal. It is found worldwide with a large range of clinical manifestations and high prevalence, which indicates that it is one of the most successful parasites of man. Serious disease due to various parasite strains occurs in congenitally infected children and in immunocompromised patients; in particular, in the case of HIV infection (Hill et al., 2005; Montoya \& Liesenfeld, 2004). Not only the severity of toxoplasmosis, but also the immune response against it, is partly determined by parasite strain (Boothroyd \& Grigg, 2002; Rodgers et al., 2005; Saeij et al., 2005).

The presence of different strains of $T$. gondii with different antigenic characteristics has been demonstrated by detailed studies with $\mathrm{mAb}$ techniques, Western blotting, isoenzyme analyses, RFLP, and random amplified polymorphic DNA PCR (RAPD-PCR) (Ware \& Kasper, 1987; Kong et al., 2003; Darde et al., 1992; Darde, 2004; Ajzenberg et al., 2005).

In this study, we aimed to evaluate possible straindependent variations of two different Toxoplasma strains, $\mathrm{RH}$ and $\mathrm{RH}$ Ankara, in the detection of anti-Toxoplasma

Abbreviations: IFA, indirect immunofluorescence antibody; WB, Western blotting.
IgG antibodies by ELISA, and indirect immunofluorescence antibody (IFA) and Western blotting (WB) techniques.

\section{METHODS}

Subjects. The study group for investigation of anti-Toxoplasma IgG antibodies consisted of 72 patients with suspicion of toxoplasmosis who presented to the Adnan Menderes University Medical Faculty Parasitology Department in 2001-2002. Ten healthy subjects negative for toxoplasma infection were used as negative controls in serological tests. Approximately $6 \mathrm{ml}$ venous blood was taken from each patient and centrifuged for separation of serum. Sera were kept at $-20{ }^{\circ} \mathrm{C}$ until the serological tests were performed. Using antigens from each strain, in-house ELISA, IFA and WB tests were performed for each serum sample separately.

Antigens. T. gondii antigens were prepared from tachyzoites of the RH and RH Ankara (TRH) strains. The TRH strain, which was isolated from mice infected with intestinal extracts of stray cats in Ankara city, is considered to be the main strain of toxoplasmosis in Turkey. This strain belongs to the type 1 group, since tachyzoites of the strain kill intraperitoneally infected mice in 4-6 days (Degerli et al., 2003; Doskaya et al., 2006).

For antigen preparation, tachyzoites of the $\mathrm{RH}$ and TRH strains were harvested from the peritoneal cavities of previously infected BALB/c mice. After washing three times in PBS, the tachyzoites were used as antigen in an IFA test (Ertug et al., 2000a). After dilution at a concentration of $10^{5} \mathrm{ml}^{-1}$ in PBS, tachyzoites were fragmented with $1 \%$ SDS and centrifuged at 10000 r.p.m. and $4{ }^{\circ} \mathrm{C}$ for $30 \mathrm{~min}$. The supernatants were used as antigen in ELISA and WB tests. 
ELISA. In-house ELISA was performed as described previously (Ertug et al., 2000a, b). The optimal concentration of the antigens for ELISA was determined using the checkerboard method. Each serum was examined twice. Results were determined for each serum by calculating the mean $A_{405}$ value for duplicate wells. A positive result was estimated as any value higher than three standard deviations above the mean $A_{405}$ obtained from the 10 sera of the negative control serum group.

IFA test. The in-house IFA test was performed as described previously (Ertug et al., 2000a). Briefly, serial serum dilutions starting from $1: 16$ were prepared in PBS and dropped on wells of microscope slides covered with $T$. gondii tachyzoites. Fluorescein-labelled anti-human IgG (Sigma) was used as conjugate. Results were evaluated under the fluorescence microscope.

Immunoblotting. Soluble antigen of each strain was fractionated by SDS-PAGE and the in-house WB test was performed as described previously (Ertug et al., 2000b). Briefly, following separation (10\% separation gel, $5 \%$ stacking gel), T. gondii antigens (RH or TRH) were transferred electrophoretically onto $0.45 \mu \mathrm{m}$ pore-size nitrocellulose membranes, blocked with $3 \%$ casein and cut into strips. Strips were incubated for $1 \mathrm{~h}$ with sera and diluted $1 / 100$ in Trisbuffered saline, and protein recognition by the sera was revealed by incubation with anti-human IgG-alkaline phosphatase conjugate (Sigma) and then with the chromogenic substrate nitroblue tetrazolium/5-bromo-4-chloro-3-indolyl phosphate (NBT/BICP). The molecular masses of the bands were evaluated by comparison with a molecular mass marker (Bio-Rad) that was processed simultaneously.

\section{RESULTS AND DISCUSSION}

Of the 72 patients, 36 had anti-T. gondii IgG antibodies, detected by ELISA and IFA tests prepared with TRH strain antigen, and in addition these 36 patients had similar results with the tests performed with RH strain antigen. Twentyfive patients had low titres of antibody (IFA IgG $\leqslant 1 / 128$; ELISA IgG $\leqslant 1 / 1024$ ) and 11 had high titres of antibody (IFA IgG $>1 / 128$; ELISA IgG $>1 / 1024)$. No antibody responses were detected with TRH or RH strain antigen in the remaining 36 patient sera. All seropositive patients detected with ELISA and IFA tests also had IgG responses with WB. Similarly, seronegative patients did not show an antigenic response with WB.

The WB patterns obtained with TRH and RH strain antigens differed substantially. Fourteen different bands were obtained by using the $\mathrm{RH}$ strain antigen, ranging from 17 to $105 \mathrm{kDa}(17,22,25,30,32,34,35,38,43,48,60,70,80$ and $105 \mathrm{kDa})$. Each serum from seropositive patients recognized 8-14 of these proteins. When TRH strain antigen was used in $\mathrm{WB}$, four additional bands $(41,52,54$ and $58 \mathrm{kDa})$ were observed. While 30 and $38 \mathrm{kDa}$ molecular mass bands were darker in the blotting with the RH strain, an $80 \mathrm{kDa}$ molecular mass band was darker with the TRH strain. Representative bands of WB with $\mathrm{RH}$ and TRH antigens are shown in Fig. 1.

In biological and epidemiological studies, three main genotypes are generally recognized in the $T$. gondii population, types 1, 2 and 3 (Saeij et al., 2005; Darde et al., 1992; Howe \& Sibley, 1995). From a phylogenetic point of view, types 2 and

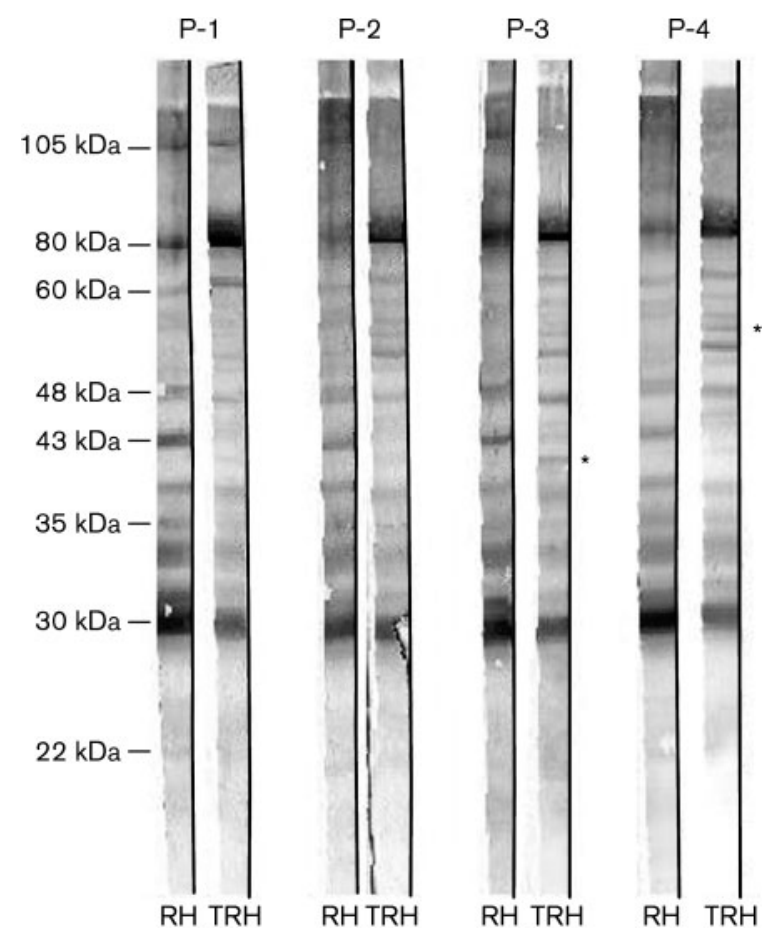

Fig. 1. Representative $\lg G$ bands of sera from four patients (P-1 to $\mathrm{P}-4)$ in WB with $\mathrm{RH}$ and $\mathrm{TRH}$ antigens. *Additional bands seen with TRH antigen.

3 belong to the same clonal group. Findings in a study of 106 isolates have suggested that type 1 isolates are significantly more often associated with human congenital toxoplasmosis than with animal infection or reactivation of chronic infections in patients with AIDS (Howe \& Sibley, 1995). When the virulent Toxoplasma strains are investigated according to phenotypic properties, significant differences are found between strains, which suggests that genetic variations can be found within this group (Appleford \& Smith, 1997). All the Toxoplasma strains are morphologically similar, and when the immune responses are examined serologically, all of them give rise to the same pattern of response (Howe \& Sibley, 1995; Dubey, 1998). In this study, we observed that the use of antigens of two different strains did not affect the results of ELISA and IFA tests. Strainspecific antigenic variability among various strains of $T$. gondii has been investigated by WB (Ware \& Kasper, 1987; Weiss et al., 1988; Appleford \& Smith, 2000). Eight antigenic bands ranging between 26 and $105 \mathrm{kDa}$ have been reported as important in differentiation of Toxoplasma strains. Bands of molecular masses 55, 71 and $130 \mathrm{kDa}$ have been observed only with the RH strain, whereas bands of $24 \mathrm{kDa}$ with the $\mathrm{C}_{56}$ strain, and 21 and $91 \mathrm{kDa}$ with the $\mathrm{RH}$ and $\mathrm{T}_{100}$ strains, have been observed. Also, the detection of different antigenic bands in two different toxoplasmosis outbreaks has shown that different strains of $T$. gondii caused the outbreaks (Weiss et al., 1988). In this study, 14 different bands of molecular masses ranging from 17 to $105 \mathrm{kDa}$ were 
obtained by using RH strain antigen. When TRH strain antigen was used in WB, three additional bands with molecular masses ranging between 48 and $60 \mathrm{kDa}$ were observed. Findings in an earlier study that included three different strains revealed that 30-33, 21 and $25 \mathrm{kDa}$ molecular mass bands were common to all the strains, but qualitative and quantitative differences were detected (Appleford \& Smith, 2000 ). In our study, the $30 \mathrm{kDa}$ molecular mass band was darker in the blotting with the RH strain antigen, similar to the results of Appleford \& Smith (2000). However, with the TRH strain antigen, an $80 \mathrm{kDa}$ molecular mass band was observed to be darker than the other bands.

It has been reported that as the antibody titres increase, the darkness of the bands seen in WB also increases (Ware \& Kasper, 1987; Cazabonne et al., 1994). We also observed that, as antibody response increased in conventional serologic tests, the number and darkness of the bands seen in WB increased. A further study with serological follow up of seropositive subjects could more clearly illustrate a direct correlation between antibody response and qualitative and/ or quantitative changes in WB.

In conclusion, the employment of antigens from the two different strains did not affect the results of ELISA or IFA tests, because of the common antigenic epitopes. On the other hand, the demonstrated differences in antigenic profiles suggest that $\mathrm{WB}$ is a valuable test for differentiation of Toxoplasma strains. Further analysis of the antigenic determinants recognized by humoral and cellular immune responses may have important implications for diagnosis, therapy and vaccination strategies.

\section{REFERENCES}

Ajzenberg, D., Dumetre, A. \& Darde, M. L. (2005). Multiplex PCR for typing strains of Toxoplasma gondii. J Clin Microbiol 43, 1940-1943.

Appleford, P. J. \& Smith, J. E. (1997). Toxoplasma gondii: the growth characteristics of three virulent strains. Acta Trop 65, 97-104.

Appleford, P. J. \& Smith, J. E. (2000). Strain and stage specific variation in Toxoplasma gondii antigens. Int J Parasitol 30, 1187-1191.

Boothroyd, J. C. \& Grigg, M. E. (2002). Population biology of Toxoplasma gondii and its relevance to human infection: do different strains cause different disease? Curr Opin Microbiol 5, 438-442.

Cazabonne, P., Bessieres, M. H. \& Seguela, J. P. (1994). Kinetics study and characterisation of target excreted/secreted antigens of immunoglobulin G, M, A and $\mathrm{E}$ antibodies from mice infected with different strains of Toxoplasma gondii. Parasitol Res 80, 58-63.

Darde, M. L. (2004). Genetic analysis of the diversity in Toxoplasma gondii. Ann Ist Super Sanita 40, 57-63.

Darde, M. L., Bouteille, B. \& Pestre-Alexandre, M. (1992). Isoenzyme analysis of 35 Toxoplasma gondii isolates and the biological and epidemiological implications. J Parasitol 78, 786-794.

Degerli, K., Kilimcioglu, A. A., Kurt, O., Tamay, A. T. \& Ozbilgin, A. (2003). Efficacy of azithromycin in a murine toxoplasmosis model, employing a Toxoplasma gondii strain from Turkey. Acta Trop 88, 45-50.

Doskaya, M., Degirmenci, A., Cicek, C., Ak, M., Korkmaz, M., Guruz, Y. \& Uner, A. (2006). Behaviour of Toxoplasma gondii RH Ankara strain tachyzoites during continuous production in various cell lines. Parasitology 132, 315-319.

Dubey, J. P. (1998). Toxoplasmosis. In Topley and Wilson's Microbiology and Microbial Infections, pp. 303-318. Edited by F. E. G. Cox, J. P. Kreier \& D. Wakelin. New York: Oxford University Press.

Ertug, S., Uner, A., Aksoy, U., Gunduz, C. \& Guruz, Y. (2000a). Correlation between ELISA, IFA and IHA techniques in the diagnosis of acute toxoplasmosis. Acta Parasitologica Turcica 24, 4-8.

Ertug, S., Uner, A., Altıntas, N., Yolasıgmaz, A. \& Petersen, E. (2000b). Comparison of ELISA and Western blotting methods for the detection of IgG antibodies against Toxoplasma gondii. Acta Parasitologica Turcica 24, 17-21.

Hill, D. E., Chirukandoth, S. \& Dubey, J. P. (2005). Biology and epidemiology of Toxoplasma gondii in man and animals. Anim Health Res Rev 6, 41-61.

Howe, D. K. \& Sibley, L. D. (1995). Toxoplasma gondii comprises three clonal lineages: correlation of parasite genotype with human disease. J Infect Dis 172, 1561-1566.

Kong, J. T., Grigg, M. E., Uyetake, L., Parmley, S. \& Boothroyd, J. C. (2003). Serotyping of Toxoplasma gondii infections in humans using synthetic peptides. J Infect Dis 187, 1484-1495.

Montoya, J. G. \& Liesenfeld, O. (2004). Toxoplasmosis. Lancet 363, 1965-1976.

Rodgers, L., Wang, X., Wen, X., Dunford, B., Miller, R. \& Suzuki, Y. (2005). Strains of Toxoplasma gondii used for tachyzoite antigens to stimulate spleen cells of infected mice in vitro affect cytokine responses of the cells in the culture. Parasitol Res 97, 332-335.

Saeij, J. P., Boyle, J. P. \& Boothroyd, J. C. (2005). Differences among the three major strains of Toxoplasma gondii and their specific interactions with the infected host. Trends Parasitol 21, 476-481.

Ware, P. L. \& Kasper, L. H. (1987). Strain-specific antigens of Toxoplasma gondii. Infect Immun 55, 778-783.

Weiss, L. M., Udem, S. A., Tanowitz, H. \& Wittner, M. (1988). Western blot analysis of the antibody response of patients with AIDS and Toxoplasma encephalitis: Antigenic diversity among Toxoplasma strains. J Infect Dis 157, 7-13. 